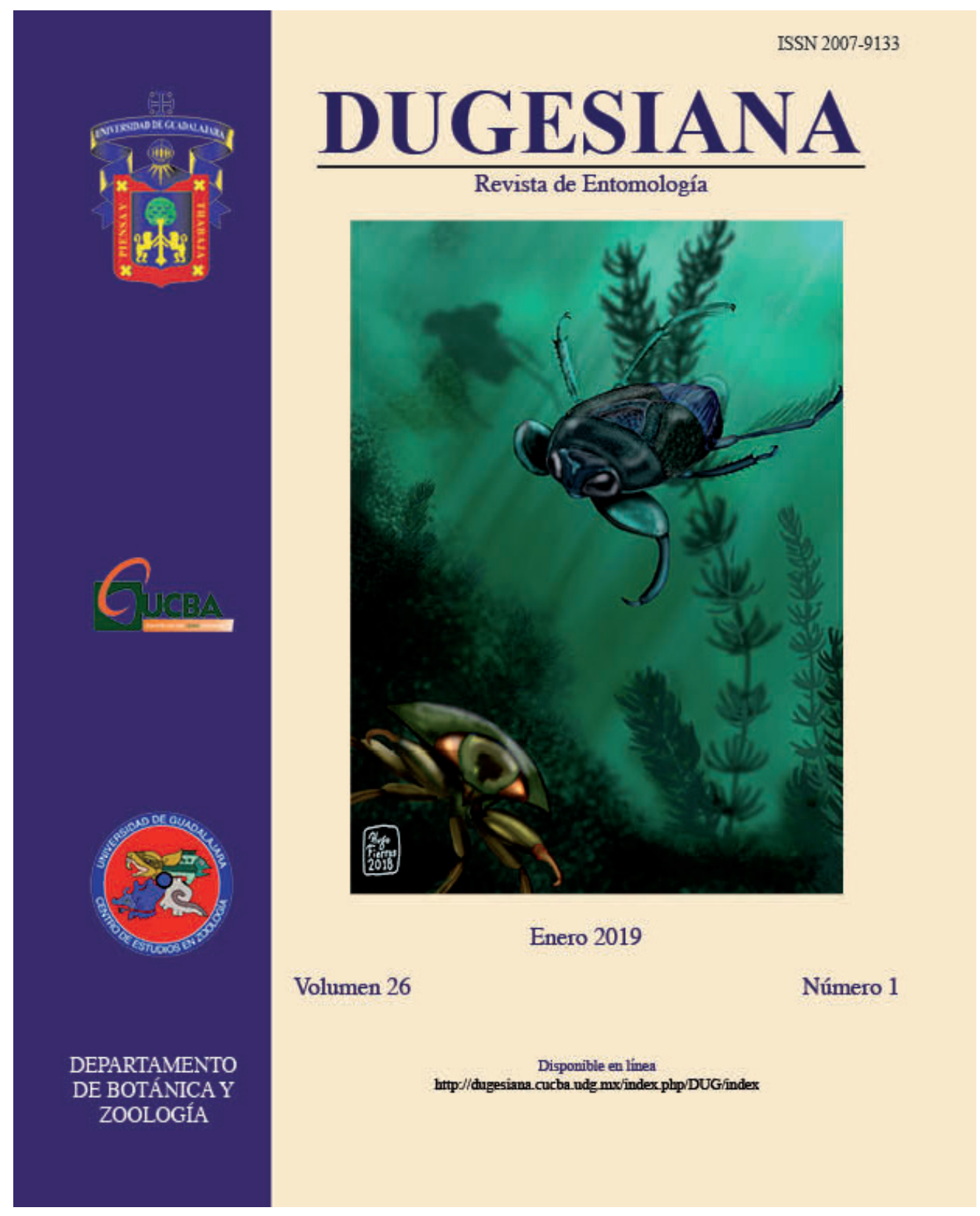

DUGESIANA, Año 26, No.1, 2019 (primer semestre de 2019) es una publicación Semestral editada por la Universidad de Guadalajara, a través del Centro de Estudios en Zoología, por la División de Ciencias Biológicas y Ambientales, CUCBA. Km. 15.5 carr. a Nogales, Predio Las Agujas s/n, Nextipac, C.P. 45100. Zapopan, Jalisco, México. Tel. 37771150, http://dugesiana.cucba.udg.mx/index.php/DUG/index, glenusmx@gmail.com, Editor responsable: José Luis Navarrete Heredia. Reserva de Derechos al Uso Exclusivo 04-2009-062310115100-203, ISSN: 2007-9133, otorgados por el Instituto Nacional del Derecho de Autor. Responsable de la última actualización de este número: José Luis Navarrete Heredia, Editor y Ana Laura González-Hernández, Asistente Editorial. Fecha de la última modificación 1 de enero de 2019, con un tiraje de un ejemplar.

Las opiniones expresadas por los autores no necesariamente reflejan la postura del editor de la publicación.

Queda estrictamente prohibida la reproducción total o parcial de los contenidos e imágenes de la publicación sin previa autorización de la Universidad de Guadalajara. 


\section{Nuevos registros de hormigas (Hymenoptera: Formicidae) en la Isla María Cleofas, Golfo de California, México}

\section{New records of ants (Hymenoptera: Formicidae) from María Cleofas Island, Gulf of California, Mexico}

\section{Fabio Germán Cupul-Magaña ${ }^{1^{*}}$ y Miguel Vásquez-Bolaños ${ }^{2}$}

${ }^{1}$ Centro Universitario de la Costa, Universidad de Guadalajara. Av. Universidad 203, Delegación Ixtapa, C.P. 48280, Puerto Vallarta, Jalisco, México, fabiocupul@gmail.com; ${ }^{2}$ Centro de Estudios en Zoología, Departamento de Botánica y Zoología, Centro Universitario de Ciencias Biologías y Agropecuarias, Universidad de Guadalajara. Apdo. Postal 134, C. P. 45100, Zapopan, Jalisco, México, mvb14145@hotmail.com

\section{RESUMEN}

La mirmecofauna de la Isla María Cleofas, una de las cuatro áreas insulares de la Reserva de la Biosfera del archipiélago de las Islas Marías en el Golfo de California, México, es poco conocida. En este trabajo se registran por primera vez para la isla a Camponotus atriceps, C. rectangularis, Leptogenys ixta, Pseudomyrmex elongatus y Solenopsis geminata. Asimismo, la documentación fotográfica previa de Atta mexicana en la localidad, fue confirmada por la revisión de especímenes.

Palabras clave: Islas Marías, lista de especies, mirmecofauna, Nayarit.

\section{ABSTRACT}

The myrmecofauna from María Cleofas Island, one of the four island of the Islas Marías archipelago Biosphere Reserve in the Gulf of California, Mexico, is poorly known. In this work, Camponotus atriceps, C. rectangularis, Leptogenys ixta, Pseudomyrmex elongatus and Solenopsis geminata were recorded for the first time from the island. Also, the previous photographic documentation of Atta mexicana in the locality, was confirmed by the examination of specimens.

Key words: Checklist, Marías Islands, myrmecofauna, Nayarit.

La mirmecofauna de la Isla María Cleofas, una de las cuatro áreas insulares de la Reserva de la Biosfera del archipiélago de las Islas Marías en el Golfo de California, México, es poco conocida. Los únicos registros publicados son de los mirmicinos Monomorium cyaneum Wheeler, 1914, presente desde el sur de los Estados Unidos de América hasta el centro de México (DuBois 1986; VásquezBolaños 2011; AntWeb 2018a), y Pheidole dwyeri Gregg, 1969, solo conocida para la isla (Gregg 1969; Wilson 2003); además del dorilino Neivamyrmex nigrescens (Cresson, 1872), de amplia distribución en los Estados Unidos de América y México (Watkins 1982; Vásquez-Bolaños 2011; AntWeb 2018b). Adicionalmente, en la plataforma de ciencia ciudadana Naturalista, desarrollada por la Comisión Nacional para el Conocimiento y Uso de la Biodiversidad (CONABIO) en colaboración con iNaturalist, se pueden consultar fotografías que documentan para la isla otras dos especies de hormigas: Atta mexicana Smith, 1858 (Naturalista-CONABIO 2018a) y Camponotus planatus Roger, 1863 (Naturalista-CONABIO 2018b), ambas de amplia distribución en las regiones Neártica y Neotropical (AntWiki 2015, 2017).

La isla María Cleofas $\left(21^{\circ} 18^{\prime} 53.45^{\prime \prime} \mathrm{N}-106^{\circ} 14^{\prime} 59.86^{\prime \prime}\right.$ O) se ubica a $132 \mathrm{~km}$ de la costa de San Blas, Nayarit, tiene una superficie de $1,882.973493$ ha y su vegetación representativa es el bosque tropical subcaducifolio (CONABIO 2007). Los artrópodos no son mencionados en el Plan de Manejo de la Reserva Biosfera del archipiélago de las Islas Marías (CONABIO 2007), por lo que este trabajo tiene como objetivo contribuir a su conocimiento con el registro de seis especies de formícidos.

\section{MATERIAL Y MÉTODOS}

Los ejemplares se recolectaron con pinzas en los meses de abril y agosto de 2018, a partir de búsquedas visuales dentro del bosque tropical subcaducifolio conservado. Se preservaron en alcohol al $70 \%$ y depositaron en la Colección Entomológica del Centro de Estudios en Zoología, del Centro Universitario de Ciencias Biológicas y Agropecuarias de la Universidad de Guadalajara (CZUG) en Zapopan, Jalisco. Para su identificación se utilizaron los trabajos de Mackay y Mackay (1989, 2004), Ward (1989), Gonçalves (1942), Trager (1991), Lattke (2011), así como Pacheco y Mackay (2013).

\section{RESULTADOS Y DISCUSIÓN}

El material examinado, recolectado en el suelo (no se revisó la vegetación), correspondió a seis especies incluidas en seis géneros y cuatro subfamilias (Cuadro 1). De estas especies, Camponotus atriceps (Fig. 1), C. rectangularis (Fig. 2), Leptogenys ixta (Fig. 3), Pseudomyrmex elongatus (Fig. 4) y Solenopsis geminata (Fig. 5), se registran por primera vez para la Isla Cleofas. Por su parte, la recolección y revisión de especímenes de $A$. mexicana (Fig. 6), corrobora su documentación fotográfica previa en la localidad (Naturalista-CONABIO 2018a).

La mirmecofauna de la Isla Cleofas tiene como fuente de recursos biológicos o de especies de hormigas, al igual que lo observado en otras islas del Golfo de California, a las áreas continentales adyacentes (Boulton y Ward 2002). Así, todas las especies observadas hasta el momento, salvo $P$. $d$ wyeri que es endémica de la isla, son conocidas tanto para la península de Baja California como para Jalisco o 
Nayarit (Vásquez-Bolaños 2011). Ahora, en cuanto a los mecanismos que pueden ser utilizados por las hormigas para llegar a las islas, se ha documentado que existen tres principales: a través vuelos de apareamiento de reproductores, por navegación sobre materiales flotantes o "rafting" y dispersión asistida por los humanos (Morrison 2016)

Asimismo, sobre el número de especies de hormigas endémicas de algunas islas del Golfo de California, Boulton y Ward (2002) comentan que una recolección intensiva en el continente probablemente reducirá este número aún más, ya que casi todas las especies se conocen para las regiones aledañas. Por lo tanto, la condición de endemismo insular de $P$. $d$ wyeri podría modificarse en un futuro al incrementarse los muestreos en el territorio de los estados vecinos; sin embargo, esta condición podría no cambiar, por el hecho de que han pasado casi 40 años de su descubrimiento y aún no hay evidencia de su presencia en la región continental.

\section{AGRADECIMIENTOS}

A la Comisión Nacional de Seguridad (CNS), Secretaria de Medio Ambiente y Recursos Naturales (SEMARNAT), Secretaria de Marina (SEMAR), Complejo penitenciario federal Islas Marías y a la Asociación Civil Protección y Restauración de Islas y Zonas Naturales (PROZONA, AC.), por su apoyo para la realización de este trabajo. A Ismael E. Huerta de la Barrera, U. Sebastián Flores Guerrero y Armando H. Escobedo Galván por su apoyo en campo. A los dos revisores anónimos por sus valiosos comentarios.

\section{LITERATURA CITADA}

AntWeb. 2018a. Species: Monomorium cyaneum Wheeler, 1914. AntWeb v7.34.4. https://www.antweb.org/description.do? genus $=$ monomorium $\&$ species $=$ cyaneum $\&$ rank $=$ species. Fecha de consulta: 28 de agosto de 2018.

AntWeb. 2018b. Species: Neivamyrmex nigrescens (Cresson, 1872). AntWeb v7.34.4. https://www.antweb.org/description. do rank $=$ species $\&$ genus $=$ neivamyrmex $\&$ name $=$ nigrescens. Fecha de consulta: 3 de septiembre de 2018.

AntWiki. 2015. Atta mexicana. http://www.antwiki.org/wiki/ Atta_mexicana. Fecha de consulta: 25 de agosto de 2018.

AntWiki. 2017. Camponotus planatus. http:/www.antwiki.org/ wiki/Camponotus_planatus. Fecha de consulta: 28 de agosto de 2018.

Boulton, A.M. and P.S. Ward. 2002. The biological scene: Ants. (pp. 112-128). In: Case, T.J., M.L. Cody and X. Ezcurra (Eds.). A new island biogeography of the Sea of Cortés. Oxford University Press, Oxford.

CONABIO. 2007. Programa de conservación y manejo Reserva de la Biosfera Islas Marías, México. SEMARNAT-CONANP, México.

Recibido: 4 de octubre de 2018

Aceptado: 31 de octubre de 2018

Primero en línea: 26 de noviembre 2018

Online First Publication: 26th November 2018
DuBois, M.B. 1986. A revisión of the native new world species of the ant genus Monomorium (minimum group) (Hymenoptera: Formicidae). The University of Kansas Science Bulletin, 53(2): 65-119.

Gonçalves, C.R. 1942. Contribuição para o conhecimento do gênero Atta Fabr., das formigas saúvas. Boletim da Sociedade Brasileira de Agronomia, 5(3): 333-358.

Gregg, R.E. 1969. New species of Pheidole from Pacific coast islands (Hymenoptera: Formicidae). Entomological News, 80: 93-101.

Lattke, J.E. 2011. Revision of the New World species of the genus Leptogenys Roger (Insecta: Hymenoptera: Formicidae: Ponerinae). Arthropod Systematics \& Phylogeny, 69(3): 127-264.

Mackay, W.P. y E.E. Mackay. 1989. Clave de los géneros de hormigas en México (Hymenoptera: Formicidae). (pp. 1-82) In: Memoria del II Simposio Nacional de Insectos Sociales. Sociedad Mexicana de Entomología, Oaxtepec, México.

Mackay, W. y E. Mackay. 2004. Especies de Camponotus de México. https://www.utep.edu/leb/ants/Mexicacamp. doc. Fecha de consulta: 28 de agosto de 2018.

Morrison, L.W. 2016. The ecology of ants (Hymenoptera: Formicidae) on islands. Myrmecological News, 23: $1-14$.

Naturalista-CONABIO. 2018a. Hormiga chicatana negra (Atta mexicana). https://www.naturalista. mx/observations/3183812. Fecha de consulta: 4 de septiembre de 2018.

Naturalista-CONABIO. 2018b. Camponotus planatus. https://www.naturalista.mx/observations/3300675. Fecha de consulta: 24 de agosto de 2018.

Pacheco, J.A. and W.P. Mackay. 2013. The systematics and biology of the New World thief ants of the genus Solenopsis (Hymenoptera: Formicidae). The Edwin Mellen Press, Lewiston.

Trager, J.C. 1991. A revision of the fire ants, Solenopsis geminata group (Hymenoptera: Formicidae: Myrmicinae). Journal of the New York Entomological Society, 99(2):141-198.

Vásquez-Bolaños, M. 2011. Lista de especies de hormigas (Hymenoptera: Formicidae) para México. Dugesiana, 18(1): 95-133.

Watkins, J.F.II. 1982. The army ants of Mexico (Hymenoptera: Formicidae: Ecitoninae). Journal of the Kansas Entomological Society, 55(2): 197-247.

Ward, P.S. 1989. Systematic studies on pseudomyrmecine ants: revision of the Pseudomyrmex oculatus and $P$. subtilissimus species groups, with taxonomic comments on other species. Quaestiones Entomologicae, 25(4): 393-468.

Wilson, E.O. 2003. Pheidole in the New World. Harvard University Press, Cambridge. 
Cuadro1. Especies de hormigas por familia, número de ejemplares (No.) por casta (o = obreras, $\mathrm{s}=$ soldados), fecha de colecta y colector, registradas en la Isla María Cleofas, Reserva de la Biosfera del archipiélago de las Islas Marías en el Golfo de California, México. (Elaborado por los autores).

\begin{tabular}{|l|l|l|l|}
\hline Especies por familias & No. & Fecha & Colector \\
\hline $\begin{array}{l}\text { Formicinae } \\
\text { Camponotus atriceps (Smith, 1858) }\end{array}$ & $70,2 \mathrm{~s}$ & 5. IV.2018 & I. Huerta \\
$\begin{array}{l}\text { Camponotus rectangularis Emery, 1890 } \\
\begin{array}{l}\text { Pseudomyrmecinae } \\
\text { Pseudomyrmex elongatus (Mayr, 1870) }\end{array}\end{array}$ & $50,3 \mathrm{~s}$ & 21. VIII.2018 & S. Flores \\
$\begin{array}{l}\text { Ponerinae } \\
\text { Leptogenys ixta Lattke, 2011 }\end{array}$ & 10 & 21.VIII.2018 & S. Flores \\
$\begin{array}{l}\text { Mirmicinae } \\
\text { Atta mexicana } \text { (Smith, 1858) } \\
\text { Solenopsis geminata } \text { (Fabricius, 1804) }\end{array}$ & 30 & S. Flores \\
& 160 & 5.IV.VIII.2018 & S. Flores \\
\end{tabular}



Figura 1-6. Ejemplares de obreras representativas de las especies de hormigas registradas en la Isla María Cleofas, Golfo de California. 1) Camponotus atriceps. 2) Camponotus rectangularis. 3) Leptogenys ixta. 4) Pseudomyrmex elongatus. 5) Solenopsis geminata. 6) Atta mexicana. (Elaborada por los autores). 